\title{
Comparative transcriptomic analysis reveals an association of gibel carp fatty liver with ferroptosis pathway
}

Xiao-Juan Zhang ${ }^{1,2,3}$, Li Zhou ${ }^{2,3}$, Wei-Jia Lu' ${ }^{2,3}$, Wen-Xuan Du, ${ }^{2,3}$, Xiang-Yuan Mi ${ }^{2,3}$, Zhi Li, ${ }^{2,3}$, Xi-Yin Li $i^{2,3}$, Zhong-Wei Wang ${ }^{2,3}$, Yang Wang ${ }^{2,3}$, Ming Duan ${ }^{2,3}$ and Jian-Fang Gui ${ }^{1,2,3^{*}}$

\begin{abstract}
Background: Fatty liver has become a main problem that causes huge economic losses in many aquaculture modes. It is a common physiological or pathological phenomenon in aquaculture, but the causes and occurring mechanism are remaining enigmatic.
\end{abstract}

Methods: Each three liver samples from the control group of allogynogenetic gibel carp with normal liver and the overfeeding group with fatty liver were collected randomly for the detailed comparison of histological structure, lipid accumulation, transcriptomic profile, latent pathway identification analysis (LPIA), marker gene expression, and hepatocyte mitochondria analyses.

Results: Compared to normal liver, larger hepatocytes and more lipid accumulation were observed in fatty liver. Transcriptomic analysis between fatty liver and normal liver showed a totally different transcriptional trajectory. GO terms and KEGG pathways analyses revealed several enriched pathways in fatty liver, such as lipid biosynthesis, degradation accumulation, peroxidation, or metabolism and redox balance activities. LPIA identified an activated ferroptosis pathway in the fatty liver. GPCR analysis confirmed that $g p x 4$, a negative regulator of ferroptosis, was significantly downregulated while the other three positively regulated marker genes, such as acs/4, tfr 1 and gcl, were upregulated in fatty liver. Moreover, the hepatocytes of fatty liver had more condensed mitochondria and some of their outer membranes were almost ruptured.

Conclusions: We reveal an association between ferroptosis and fish fatty liver for the first time, suggesting that ferroptosis might be activated in liver fatty. Therefore, the current study provides a clue for future studies on fish fatty liver problems.

Keywords: Fatty liver, Comparative transcriptome, Latent pathway, Ferroptosis, Gibel carp

\footnotetext{
* Correspondence: jfgui@ihb.ac.cn

'College of Fisheries, Huazhong Agricultural University, Wuhan 430070, China

${ }^{2}$ State Key Laboratory of Freshwater Ecology and Biotechnology, Institute of

Hydrobiology, Innovation Academy for Seed Design, Chinese Academy of

Sciences, Wuhan 430072, Hubei, China

Full list of author information is available at the end of the article
}

C C The Author(s). 2021 Open Access This article is licensed under a Creative Commons Attribution 4.0 International License, which permits use, sharing, adaptation, distribution and reproduction in any medium or format, as long as you give appropriate credit to the original author(s) and the source, provide a link to the Creative Commons licence, and indicate if changes were made. The images or other third party material in this article are included in the article's Creative Commons licence, unless indicated otherwise in a credit line to the material. If material is not included in the article's Creative Commons licence and your intended use is not permitted by statutory regulation or exceeds the permitted use, you will need to obtain permission directly from the copyright holder. To view a copy of this licence, visit http://creativecommons.org/licenses/by/4.0/ The Creative Commons Public Domain Dedication waiver (http://creativecommons.org/publicdomain/zero/1.0/) applies to the data made available in this article, unless otherwise stated in a credit line to the data. 


\section{Background}

Fish fatty liver is a common physiological or pathological phenomenon in aquaculture. The causes are complex and not well-known, and mainly include imbalance nutrition diet, environmental pollutants, physiological factors and genetic mutation [1]. Fatty liver diseases have been found in most main farmed fish, and caused many problems, such as low feed efficiency, immune response, flesh and nutritional quality effects [1-5]. The utilization of artificially formulated feeds can bring nutritional, physiological and ecological effects to fish [2, 6-9]. However, exceeding nutrition, improper artificial formulated diets and overfeeding have led to a growing concern of liver fatty problems, such as hepatocyte enlargement, lipid accumulation, steatosis, fibrosis and necrosis $[10,11]$.

Fatty liver can be mainly classified as "nutritional fatty liver" or "oxidative fatty liver" in aquaculture. Nutritional fatty liver, principally caused by imbalanced nutrition supply, is common in farmed fish and generally not a pathological symptom. It can be alleviated through adjusting diet formulation or feeding. If no effective strategy was carried on, it could be turned to the "oxidative fatty liver" or directly induced to hepatic fibrosis and necrosis, which causes irretrievable damages [1]. Oxidative stress might lead to oxidative fatty liver, which would arouse severe liver damage $[1,12]$. Overall, excess dietary energy intake and severe peroxidation can cause fish metabolic imbalance and then result in fatty livers. Some researchers suggest that many pathways, such as target-of-rapamycin complex 1 (Torc1), AMP-activated protein kinase (AMPK), transcription factor EB (TFEB), peroxisome proliferator activated receptor (PPAR), P53, nuclear erythroid 2-related factor 2 (Nrf2), c-jun Nterminal kinase (JNK), toll-like receptors (TLRs), myeloid differentiation primaryresponse protein 88 (Myd88), and nuclear factor $\mathrm{kB}$ (NF$\kappa B)$ signaling pathways, might be related to fatty liver caused by high-fat/carbohydrate or over-nutrition diet [11-17]. For example, the decreased AMPK pathway can suppress autophagy and then worsen lipotoxicity in tilapia fatty liver [11], and its activation can reduce the expression of genes related to lipogenesis in rainbow trout liver [16]. However, some results seem to be controversial. For instance, the activation of Nrf2, JNK and TLRs-Myd88$\mathrm{NF}-\mathrm{kB}$ signaling pathways could lead to inflammation and worsen tilapia liver injury [12], while cAMP-JNK/NF-kBcaspase signaling pathway could protect the fatty liver tissues from more serious damage though regulating the hemostasis phosphorylation of JNK protein in Japanese seabass [17]. JNK and NF-KB signaling pathways might play a dual role in the fish fatty liver. Therefore, the mechanism of hepatic lipid accumulation and fish fatty liver are remaining enigmatic. In addition, too many affected pathways were identified and few studies had explored which key pathway was associated with fish fatty live.
Gibel carp (Carassius gibelio) is one of the most important aquaculture species in China [18-23] and the production yields in China have increased to 2,755,632 tons in 2019 [24]. In lotus-fish farming ponds, we found some individuals of gibel carp had fatty liver. To find out the cause, we first analyzed the liver histological structures and lipid accumulation of normal liver and fatty liver. Then, we conducted comparative transcriptomic analysis between, and identified a pathway of ferroptosis that was significantly activated in fatty liver. Finally, we confirmed that ferroptosis was activated in fatty liver by qPCR analysis and mitochondria morphological observation. Our current study establishes an association between ferroptosis and fish liver fatty, which provides a clue for future studies on fish liver fatty problems.

\section{Results}

\section{Morphological changes in fatty liver}

The morphology of fatty liver showed obviously different from that of normal liver (Fig. 1a). The whole liver was more hypertrophic and the hepatocytes of fatty liver were more enlarged (Fig. 1b), showing less hepatocytes on an area of $10 \mu \mathrm{m}$ square (Fig. 1c) $(p<0.01)$. And then, we performed oil red $\mathrm{O}$ staining (ORO) to compare the lipid accumulation between normal liver and fatty liver. As we expected, fatty liver showed more than 2 times lipid accumulation than that in normal liver (Fig. 1d-e) $(p<0.01)$.

\section{Transcriptomic differences between normal liver and fatty liver}

The transcriptomes of six liver samples in two groups were obtained using BGISEQ-500 Iillumina sequencing plat and each sample produced an average of $10.16 \mathrm{~Gb}$ clean bases. An average of 83.42 and $69.46 \%$ clean reads were mapping to the gibel carp' genome and gene sets (Table 1).

Finally, a total of 37,077 unigenes were obtained, which included 32,679 known genes and 4398 novel genes. Principal component analysis (PCA) showed groupings between normal liver and fatty (Fig. S1a). The correlation coefficient $\left(R^{2}\right)$ is $>0.93$ in group and $<0.83$ between groups (Fig. S1b), suggesting the best-fitting regression line for the technical replicates according to standards and best practices for RNA-Seq. The results demonstrate that though there are individual differences, the two groups have a totally different transcriptional trajectory (Fig. S1) [25, 26].

\section{Enriched GO terms in fatty liver}

A total of 3480 differentially expressed genes (DEGs) were assigned with the correction of $p$-values using FDR $\leq 0.001$ (Table S1). Compared to normal, 1997 genes in fatty liver were upregulated and 1483 genes 


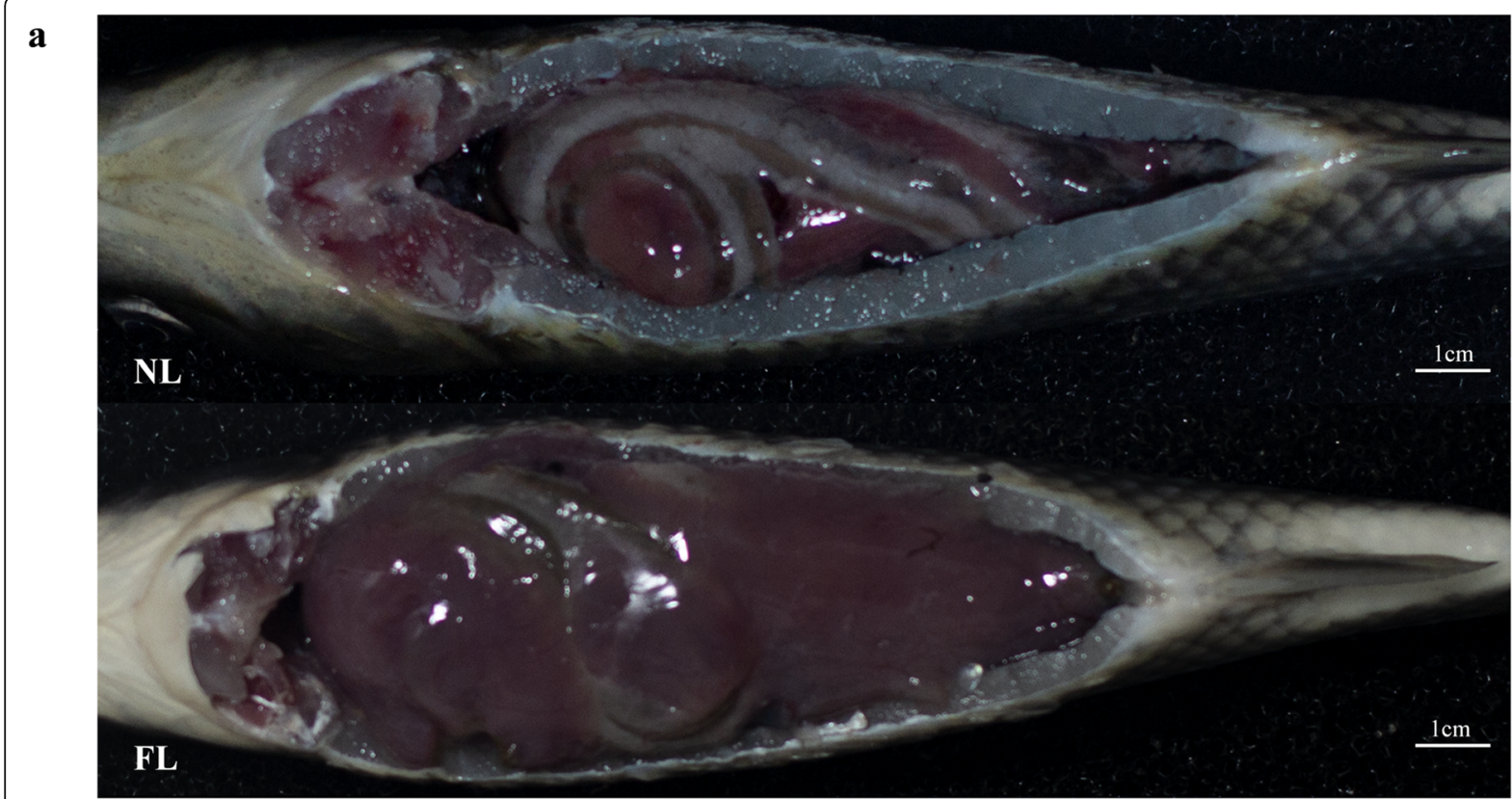

b

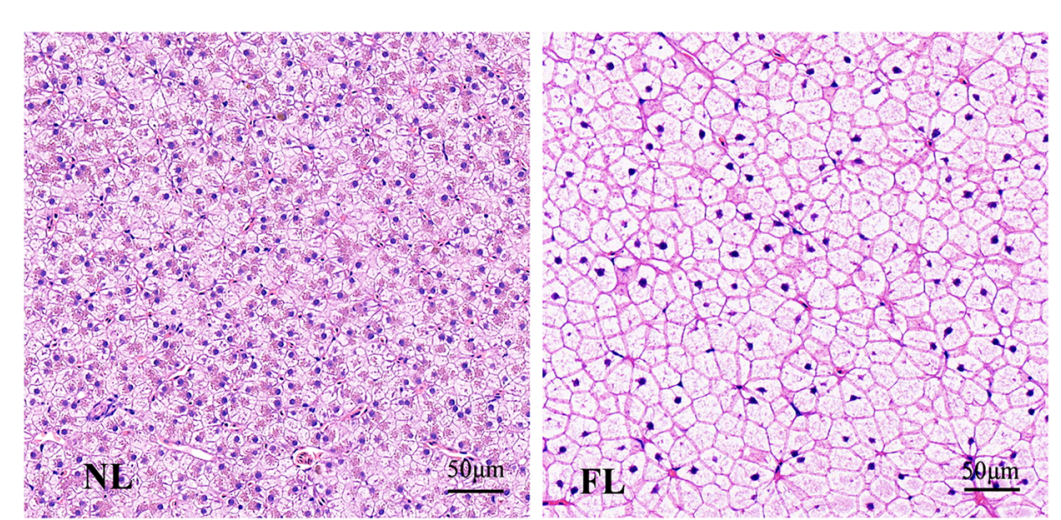

d

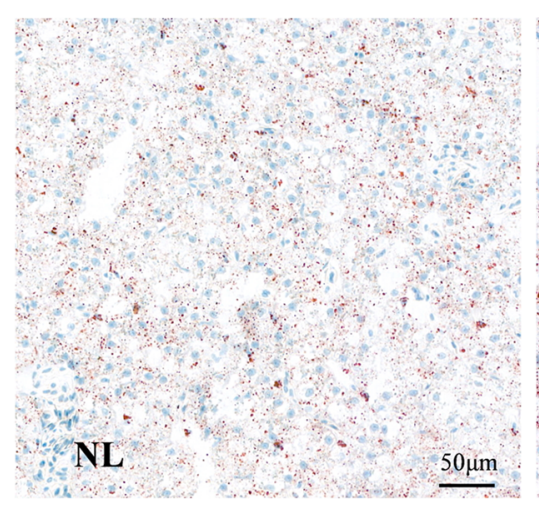

c

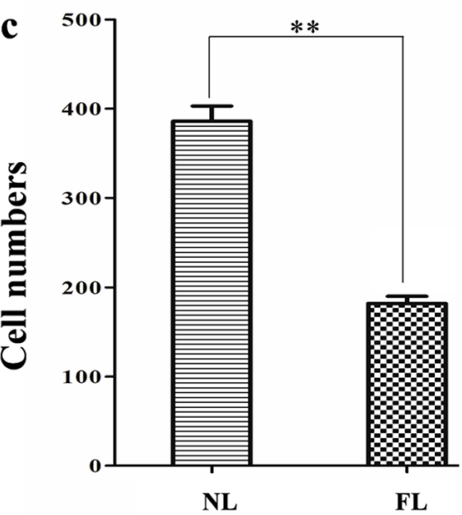

e

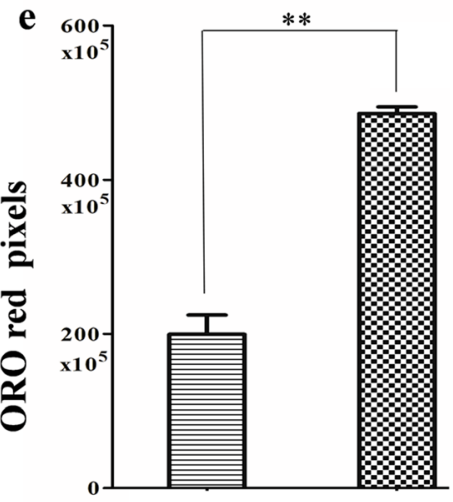

NL

Fig. 1 Histology and lipid accumulation of gibel carp normal liver and fatty liver. a Liver morphology of gibel carp. b Historical structure of liver tissues. The black column is scale bar $(50 \mu \mathrm{m})$. c Number of hepatocytes on an area of $10 \mu \mathrm{m}$ square. $\mathbf{d}$ ORO staining of liver tissues. Red color indicates lipid droplets. The black column is scale bar $(50 \mu \mathrm{m})$. e ORO red pixels $\left(X 10^{5}\right)$ in equivalent area. Asterisks stand for the significant differences between normal liver and fatty liver (**: $p<0.01)$. NL: normal liver, FL: fatty liver 
Table 1 Summary statistics of sequencing data

\begin{tabular}{llllllll}
\hline Sample & $\begin{array}{l}\text { Total Raw Reads } \\
\text { (M) }\end{array}$ & $\begin{array}{l}\text { Total Clean Bases } \\
\text { (Gb) }\end{array}$ & $\begin{array}{l}\text { Clean Reads Q20 } \\
\text { (\%) }\end{array}$ & $\begin{array}{l}\text { Clean Reads Q30 } \\
\text { (\%) }\end{array}$ & $\begin{array}{l}\text { Clean Reads } \\
\text { Ratio (\%) }\end{array}$ & $\begin{array}{l}\text { Total Mapping } \\
\text { (\%) }\end{array}$ & $\begin{array}{l}\text { Uniquely } \\
\text { Mapping (\%) }\end{array}$ \\
\hline NL-1 & 77.13 & 10.07 & 96.50 & 88.09 & 87.05 & 83.05 & 47.00 \\
NL-2 & 77.13 & 10.14 & 96.50 & 88.18 & 87.61 & 81.42 & 45.64 \\
NL-3 & 77.13 & 10.22 & 96.60 & 88.4 & 88.37 & 81.56 & 45.11 \\
FL-1 & 73.62 & 10.27 & 96.55 & 88.09 & 93.01 & 85.16 & 46.19 \\
FL-2 & 73.62 & 10.10 & 96.58 & 88.16 & 91.43 & 84.83 & 47.37 \\
FL-3 & 73.62 & 10.19 & 96.68 & 88.44 & 92.26 & 84.47 & 45.95 \\
\hline
\end{tabular}

Note: NL normal liver, FL fatty liver

were downregulated (Fig. 2a). All up or downregulated DEGs were separately annotated into 2568 GO Gene Ontology (GO) terms and 3014 GO terms, among them, as visualized in Venn's diagrams, 964 and 1410 GO terms were just associated with up or downregulated DEGs (Fig. 2b). Sixteen and $20 \mathrm{GO}$ terms were significantly enriched with the correction of q-value $\leq 0.05$ (Fig. 2 c-d).

Sixteen significantly enriched GO terms with all upregulated DEGs (Table S2), were involved in nine "biological processes" (BP), one "cellular component" (CC), six "molecular functions" (MF) (Fig. 2c). Four GO terms, "fatty acid metabolic process" (15 DEGs, GO 0006631), "fatty acid biosynthetic process" (13DEGs, GO 0006633), "unsaturated fatty acid biosynthetic process" (6 DEGs, GO 0006636), "unsaturated fatty acid metabolic process" (6 DEGs, GO 0033559), gave a direct hint that lipid synthesis or metabolism were active. Other upregulated GO terms were assigned to "fructose", "heme", "oxidoreductase activity", "hemoglobin complex", and "CoA-related activity". And these genes might play important roles in the lipid accumulation, fatty acid transport and oxidation, redox balance, or other metabolic regulatory processes $[13,27-29]$.

Twenty significantly enriched GO terms with all downregulated DEGs (Table S2), were involved in 5 BP, $9 \mathrm{CC}$, and six MF (Fig. 2d). Most of the downregulated GO terms were related to "proteasome" or "peptidase", such as "proteasome complex" (27 DEGs, GO 0000502), "proteasome core complex" (15 DEGs, GO 0005839), "proteasome accessory complex" (10 DEGs, GO 0022624), "proteasome-activating ATPase activity" (6 DEGs, GO 0036402), "peptidase complex" (31 DEGs, GO 1905368), "endopeptidase complex" (27 DEGs, GO 1905369), and "threonine-type peptidase activity" (15 DEGs, GO 0070003). Enzymes of them could catalyze biological reactions rapidly and unidirectionally regulate diverse basic cellular activities [30], suggesting some catalytic reactions might slow down in fatty liver.

\section{Significant pathways revealed by KEGG and LPIA in fatty liver}

A total of 2194 DEGs were assigned to 336 Kyoto Encyclopedia of Genes and Genomes (KEGG) pathways
[31], and some (43) of them were significantly enriched with the correction of q-value $\leq 0.05$ (Fig. 3a; Table S3) and functionally divided into six categories, including one "cellular process", one "environmental information processing", six "genetic information processing", six "diseases", 27 "metabolisms", and two "organismal systems". Five enriched pathways, such as "Ferroptosis" (29 DEGs, ko04216), "Non-alcoholic fatty liver disease (NAFLD)" (65DEGs, ko04932), "Fatty acid biosynthesis" (15DEGs, ko00061), "Fatty acid degradation" (20DEGs, ko00071), "Glycerolipid metabolism" (32DEGs, ko00561), and "Fat digestion and absorption" (32DEGs, ko04975), were directly associated with lipid biosynthesis, degradation, peroxidation, or metabolism [32, 33].

According to the LPIA method, a total of 316 KEGG pathways and $962 \mathrm{GO}$ terms with biological process classification, which two shared at least $1 \mathrm{DEG}$, were selected to conduct the pathway-pathway interaction network (Table S4). Four significant pathways were identified with LPIA_v_1.pl [34] in Perl with 1000 iterations (Table 2), such as "Porphyrin and chlorophyll metabolism" (23 DEGs, ko00860) (Fig. S2a), "Hypoxia-inducible factor1(HIF-1) signaling pathway" (49 DEGs, ko04066) (Fig. S2b), "Ferroptosis" (29 DEGs, ko04216) (Fig. 4a), and "Mineral absorption" (17 DEGs, ko04978) (Fig. S2c).

After the weight Aij was calculated at random walk, a correlational network was conducted between pathways that included 43 enriched pathways in KEGG enrichment analysis and four significant latent pathways (marked with red point) in LPIA method (Table S5). It was clearly showed that four significant latent pathways connected with each other in the network (weight scores $>0.1$ ) (Fig. 3b). The pathways "Porphyrin and chlorophyll metabolism" (23 DEGs, ko00860) (Fig. S2a) and "HIF-1 signaling pathway" (49 DEGs, ko04066) (Fig. S2b) had more connections with other pathways in KEGG enrichment analysis. Among them, excepting ZIP8/14 \& Ferritin with a up \& down expression, glutathione peroxidase 4 (gpx4), a negative regulated gene in "Ferroptosis" [35-37], was downregulated, while other DEGs in "Ferroptosis" were all upregulated (Fig. 4a). The results suggest that the expression levels of genes in 

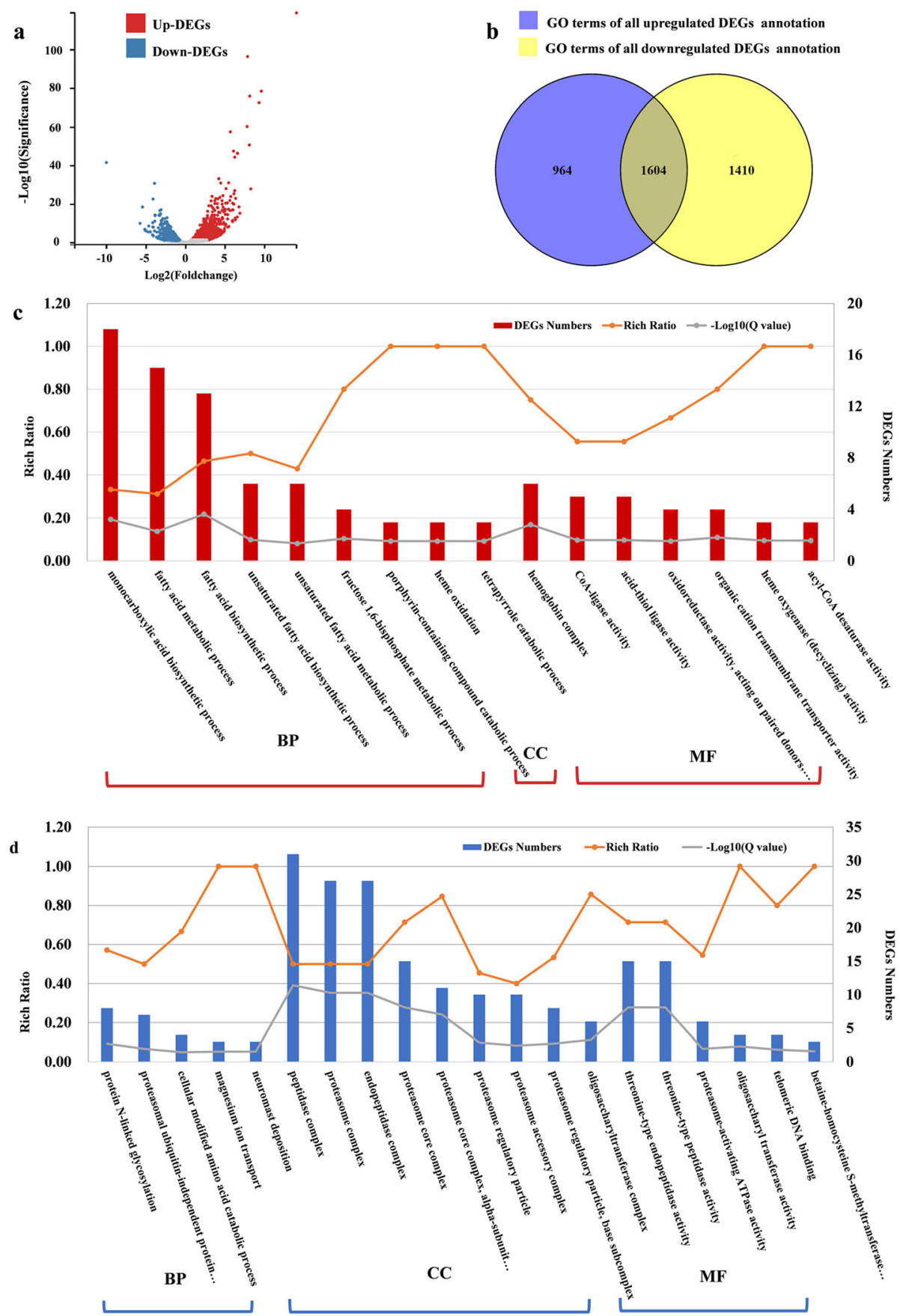

Fig. 2 DEGs and GO enrichment analysis of gibel carp normal liver and fatty liver. a Gene expression patterns between two groups. b Venn's diagrams visualize the up or downregulated DEGs associated with $\mathrm{GO}$ terms. c 16 enriched GO terms with all up- regulated DEGs (q-value $\leq 0.05$ ). d 20 enriched GO terms with all down- regulated DEGs (q-value $\leq 0.05$ ). BP: biological processes. CC: cellular component. MF: molecular functions. NL: normal liver, FL: fatty liver

pathway "Ferroptosis" might be significantly different between normal liver and fatty liver.

\section{Ferroptosis is activated in fatty liver}

To validate the different expressions of genes in "Ferroptosis" between normal liver and fatty liver, four marker genes, gpx4 [35-40], acyl-CoA synthetase long-chain family member 4 (acsl4) [41, 42], transferrin receptor 1 ( $t f r 1)$ [43], and Glutamate-cysteine ligase ( $\mathrm{gcl}$ ) [44], were selected for qPCR analysis. Consistent with the transcriptome result, gpx4 was downregulated, while the others were all upregulated (Fig. 4b). 


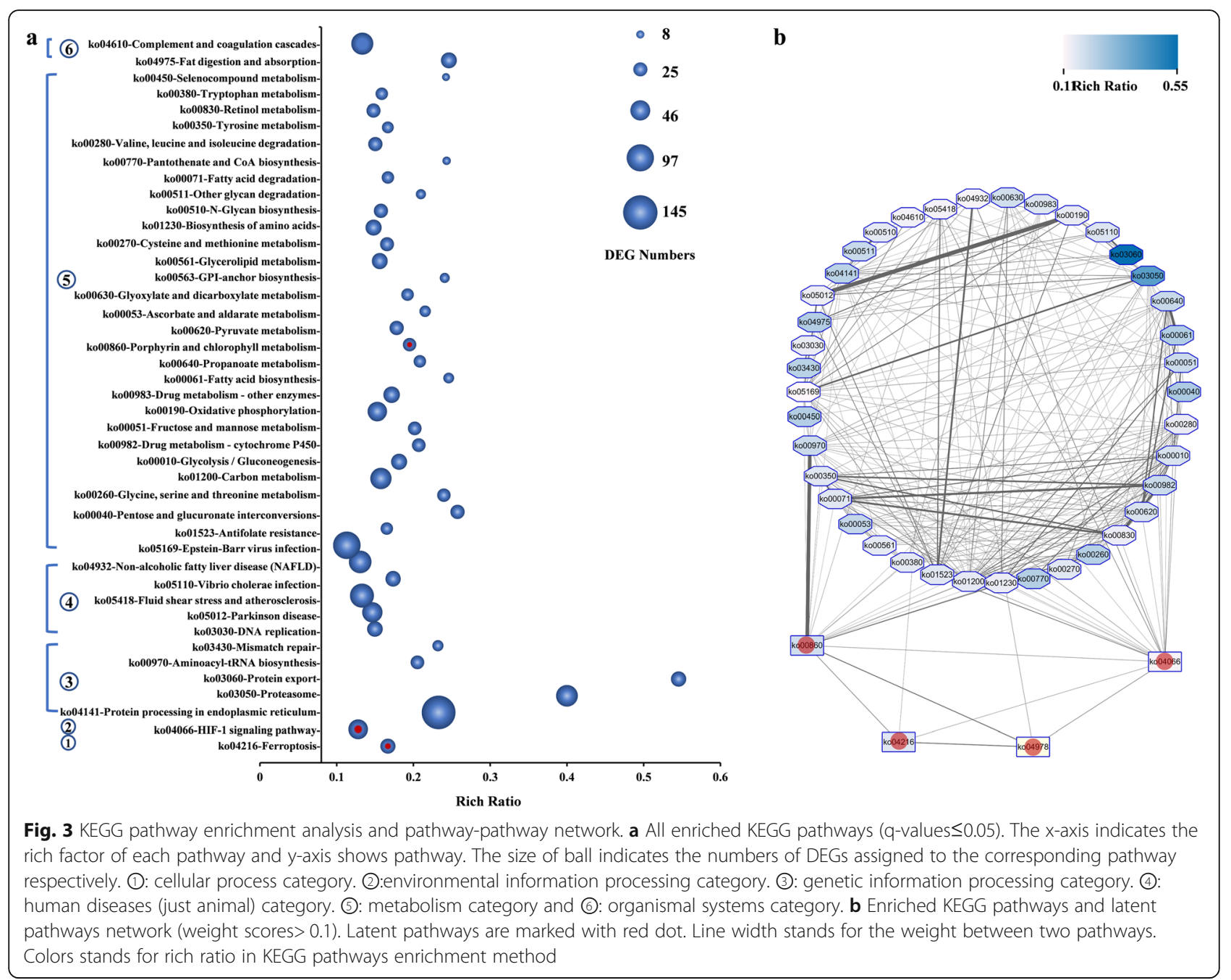

Ferroptosis can also be characterized with the morphology of mitochondria $[32,45]$. To observe the morphological differences of mitochondria between fatty liver and normal liver, we performed transmission electron microscopy analysis. There was no significant cellular dysfunction, but compared to normal liver, the mitochondria densities in fatty liver were more condensed, and some of outer mitochondrial membrane had been ruptured and seemed like single-membrane (Fig. 5).
Taken together, ferroptosis was more sensitive in the fatty liver.

\section{Discussion}

In this study, we found morphological changes in fatty liver, such as hepatocyte enlargement and lipid accumulation (Fig. 1). GO and KEGG enrichment analysis showed that activities related to lipid biosynthesis, degradation, accumulation, peroxidation or metabolism

Table 2 The significant pathways in pathway-pathway interaction network by LPIA method

\begin{tabular}{llll}
\hline Pathways & Adjusted $\boldsymbol{p}$-value & Rich Ratio $^{\mathbf{2}}$ & q-value $^{\mathbf{2}}$ \\
\hline ko00860-Porphyrin and chlorophyll metabolism & 0.00 & 0.1949 & 0.0028 \\
ko04066-HIF-1 signaling pathway & 0.00 & 0.0296 & 0.0033 \\
ko04216-Ferroptosis & 0.00 & 0.1667 & 0.0057 \\
ko04978-Mineral absorption & 0.00 & 0.1104 & $0.5570^{*}$ \\
\hline
\end{tabular}

Note: Adjusted $p$-value ${ }^{1}$ was calculated in LPIA method;

Rich Ratio ${ }^{2}$ and $q$-value ${ }^{2}$ were calculated in KEGG pathways enrichment method 


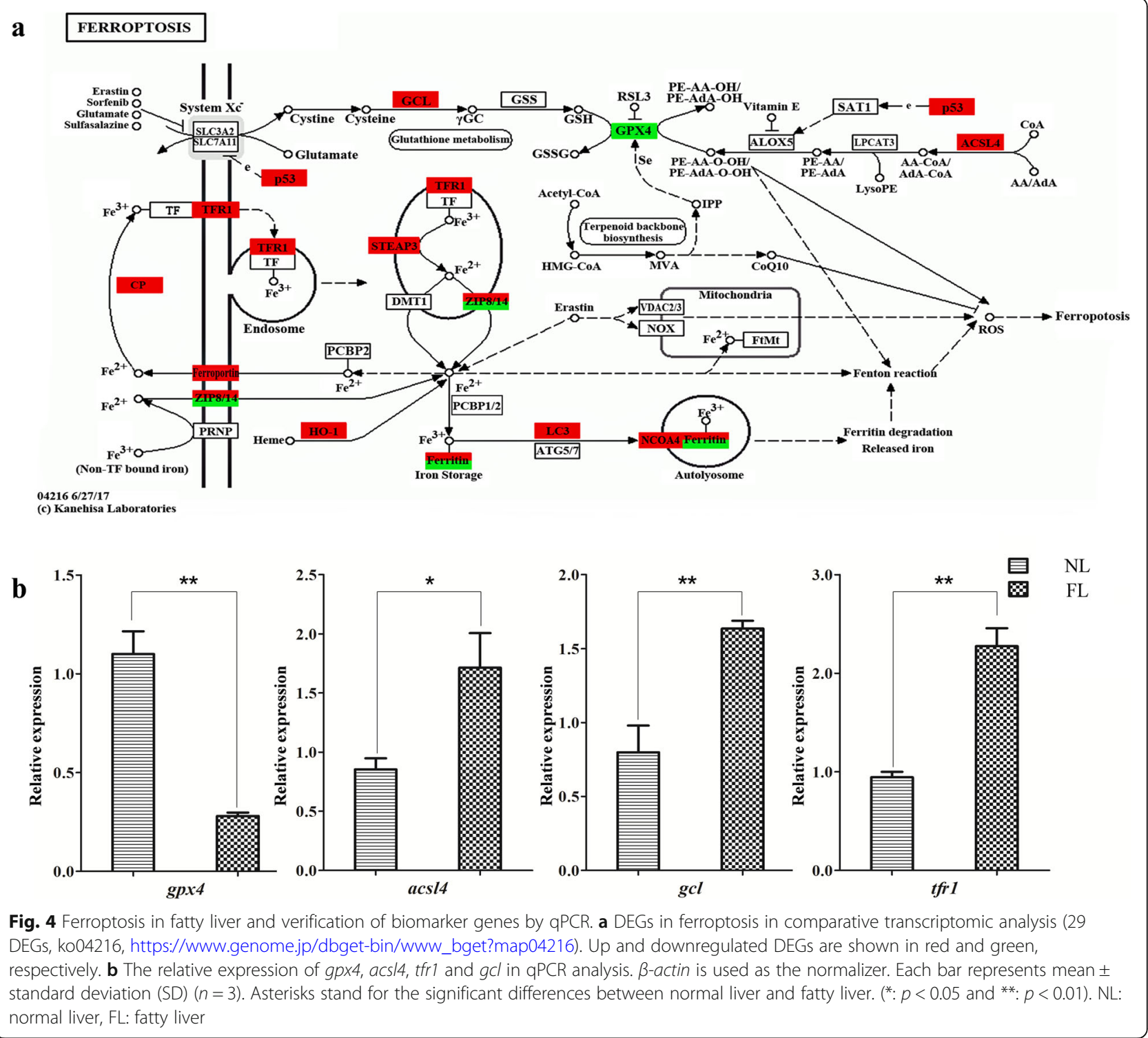

were more active in fatty liver (Fig. 2e \& Fig. 3a). Importantly, a pathway of ferroptosis was significant different between normal liver and fatty liver that might be associated with lipid-related activities (Figs. 4 \& 5), suggesting an association between ferroptosis and fish fatty liver.

Based on the current data, we suggest that a significant pathway of ferroptosis might be associated with fish fatty liver. Ferroptosis is actually an iron-catalyzed-lipid peroxidation disorder, and is related with lipid peroxidation. Previous research in fish suggested a possible link between ferroptosis and lipid. For example, the ironcatalyzed lipid peroxidation was characterized in zebrafish and cultured shrimp [46, 47]. Enzymes, such as glutathione peroxidase, could reduce the lipid peroxides in tilapia and other fish $[48,49]$. Oxidants could damage mitochondrial membrane permeability and electron transport chain integrity in zebrafish and grass carp [5052]. However, ferroptosis in fish is not clear. Now, we establish an association between ferroptosis and fatty liver through comparative transcriptomic analysis, marker gene expression and mitochondrion morphology observation in fish.

Ferroptosis is a new form of regulated cell death that depends on iron- and lipid-based reactive oxygen species (ROS), and has been implicated in both normal and pathological physiology which involves in various biological contexts of diverse species increasingly and widely $[32,35,38,53]$. It is totally different from other reported forms of cell death, such as apoptosis, autophagy, necrosis and pyroptosis [40, 53, 54], and associated with various liver problems $[38,55]$. In fish, it potentially 

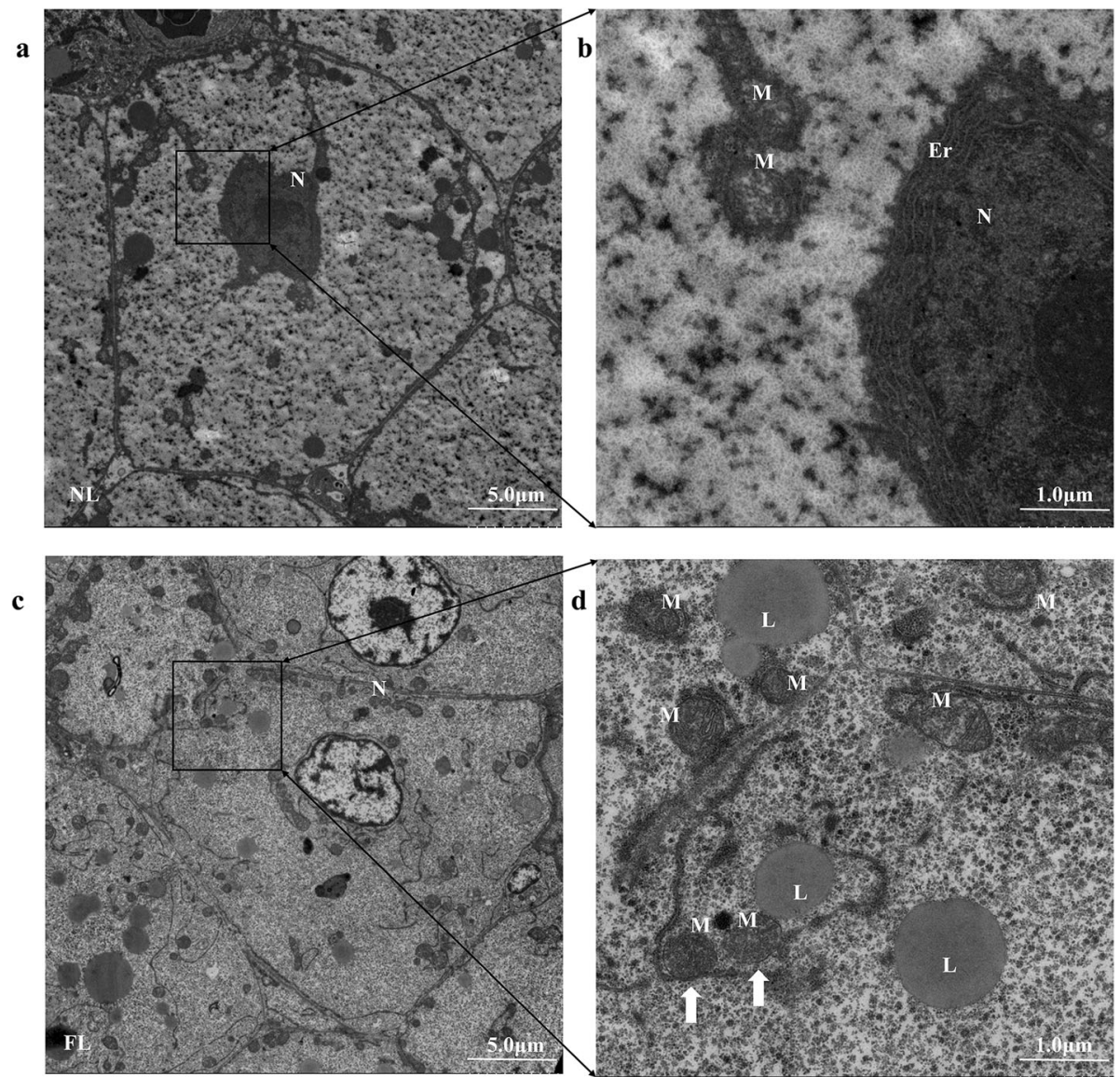

Fig. 5 Electron micrographs of hepatocytes of gibel carp normal liver and fatty liver. a-b Electron micrographs of hepatocytes in normal liver; c-d Electron micrographs of hepatocytes in fatty liver. Er: endoplasmic reticulum. L: lipid droplet. M: mitochondria. N: nucleus. The white arrows indicate the condensed mitochondria with outer membrane ruptured. The white scale bar stands for $5 \mu \mathrm{m}$ in a \& c, and $1 \mu \mathrm{m}$ in b \&d. NL: normal liver, FL: fatty liver

was responsible for the lethality of zebrafish VitEdeficient embryos [56]. It might be activated by low temperature (Nile tilapia), hypoxia (Silver sillago) stress and heavy metal (Japanese flounder) [57-59], and could be induced by Escherichia coli and then led to the red blood cells (RBCs) death of grass carp [60]. It could be checked at the gene expression level and characterized by the morphology of mitochondria [32, 35, 36, 38, 39, $41-45,61]$. Some genes in ferroptosis are classified as markers. For example, gpx4 is required for the clearance of lipid ROS. If it was inactivated or inhibited, lipid ROS would accumulate, and then induced ferroptosis [3540]. acsl4, as an essential component for ferroptosis execution, could dictate ferroptosis sensitivity. The more upregulated expression of acsl4, the more sensitive to ferroptosis [41, 42]. In this study, compared to normal liver, the relative expression of negative regulator gpx4 was significantly downregulated and the relative expression of three positive actors (acsl4, tfr 1 and $g c l$ ) were significantly upregulated, implying that fatty liver was more sensitive to ferroptosis. In addition, ferroptosis showed smaller, condensed and outer membrane ruptured of mitochondria with crista diminished or vanished in cellular morphological characteristics [62, 63]. Moreover, the GO and KEGG enrichment analysis suggest lipidrelated activities were active in fatty liver. Therefore, our results indicate that ferroptosis might be associate with liver fatty and it is different between normal liver and fatty liver.

The main method used in this study was LPIA, which was more appropriate to our study in one challenge with metabolic relevant. It could give a direct understanding to the key cellular mechanisms in biological activities $[34,64]$. In this study, four latent pathways were identified by LPIA (Table 2), including ferroptosis. Among them, the remaining three latent pathways might also connect with ferroptosis (Fig. 3b). For example, "Porphyrin and chlorophyll metabolism" pathway was associated with the energy, ion and erythroid heme synthesis regulation, especially in responding to various stresses [65, 
66]. "HIF-1 signaling" pathway was involved in cellular responses to low oxygen environments and growth factors $[67,68]$. It was noteworthy that both "Porphyrin and chlorophyll metabolism" and "HIF-1 signaling" pathways had more relations with other enriched pathways (Fig. 3b). Minerals were fundamental nutrients and mineral absorption may depend on dietary ingredient composition. Some genes in "mineral absorption pathway" were involved in iron metabolism [69, 70]. These could explain why they were all identified and suggest that ferroptosis may be the key pathway involved in fatty liver.

Overall, as lipid accumulation continued to increase, lipid peroxidation would also increase [52]. If hepatocytes could not be sufficient capacity to eliminate lipid peroxides, ferroptosis might be activated [32, 53]. Certainly, more research on the mechanism underlying ferroptosis and fish fatty liver need to be carried out. Since ferroptosis could be regulated and numbers of small molecule inhibitors, such as Vitamin E, the nature's most-efficient ferroptosis inhibitor, have been identified [32, 33, 40, 56, 71-73], which suggests new prevention strategies and promising therapies of fish fatty liver.

\section{Conclusion}

Based on detailed comparison of histological structure, lipid accumulation, transcriptomic profile, ferroptosis marker gene expression, and hepatocyte mitochondria between normal liver and fatty liver, our study reveals an association between ferroptosis and fatty liver in fish for the first time, suggesting that ferroptosis might be activated in fish liver fatty. The current study provides a clue for future studies on fish fatty liver and effective prevention strategies in fish fatty liver problems.

\section{Materials and methods}

\section{Animals and sample collection}

All experimental procedures in this study were performed in accordance with the guidelines and after approval of the Animal Care and Use Committee of Institute of Hydrobiology, Chinese Academy of Sciences (IHB, CAS, Protocol No. 2016-018). A total of 3000 juveniles of allogynogenetic gibel carp, which came from same clones, were transported from National Aquatic Biological Resource Center of Institute of IHB, CAS, which is located in Wuhan, China, to the Luo-Fu Lotus Farm, which is located in Jinggangshan, China, for lotusfish culturing. Fifty juveniles were selected randomly to weight (Table S6). Half of the juveniles was set as an overfeeding group, which was continuing feeding until without fish activities in apparent satiation two times per day. The other half was set as a control group, which was cultured without artificial feeding. All the juveniles were cultured in lotus-fish culturing ponds at a density of 150 juveniles per $667 \mathrm{~m}^{2}$. Water samples were collected from three randomly ponds three times (April 22th, June 3th, October 12th, 2019). Eight physicochemical water quality parameters (water temperature, dissolved oxygen, $\mathrm{pH}$, total nitrogen, ammonia, nitrate, nitrite and total phosphorus) were analyzed (Table S7). After 180 days, commercial fish were harvested. Fifty commercial fish of each group were selected randomly to weight (Table S6). Compared to the individuals in the control group with normal liver, most of individuals in the overfeeding group had bulge belly and randomly selected of them had fatty liver in general appearance after dissecting. Then, the liver samples of three individuals of the control group with normal liver and three individuals of the overfeeding group with fatty liver were collected randomly for histopathological observation and molecular analysis.

All fish were first totally immersed and deeply anesthetized with tricaine methanesulfonate (MS-222, 35-40 $\mathrm{mg} / \mathrm{L}$, Servivebio, Wuhan) until losing all rhythmic opercular movements for a minimum of $30 \mathrm{~min}$, then sacrificed by rapidly cutting off the spinal cord adjacent to the head. Procedures were performed to minimize fish suffering as far as possible. All sections of this study were carried out in compliance with the ARRIVE guidelines [74], and a completed ARRIVE guidelines checklist was included (Checklist S1).

\section{Histological structure, lipid accumulation and morphological observation}

Liver tissues were cut in sections and fixed in $4 \%$ paraformaldehyde (PFA) overnight at $4{ }^{\circ} \mathrm{C}$, and subsequently embedded in paraffin. One part of sections was stained with hematoxylin \& eosin (HE) (Beyotime, Suzhou) and performed as previously described [75, 76]. The CarlZeiss microscopy (Analytical \& Testing Center, IHB, CAS) was used for histological structure observation and photomicrographs.

The other sections were used for detecting the lipid droplet morphology by ORO staining according to the previous method [77, 78]. The sections were totally dehydrated with $30 \%$ sucrose, and then embedded in optimum cutting temperature (OCT) compound. After being rapidly frozen and cut in Thermo CRYOSTAR NX50 microtome at $10 \mu \mathrm{m}$ thickness, the sections were dyed with ORO (Thermo, USA) and counterstained with hematoxylin (Servivebio, Wuhan). Via the amount of ORO staining from 3 frames per biopsy, liver lipid accumulation was quantified using pixel numbers with Image $J$ [79].

The specimens for morphological observation by electron microscopy were prepared as described previously [80, 81]. Liver tissues were fixed with $2.5 \%$ glutaraldehyde for $24 \mathrm{~h}$ at $4{ }^{\circ} \mathrm{C}$, and in $1 \%$ osmium tetroxide (OsO4) for $2 \mathrm{~h}$ at $4{ }^{\circ} \mathrm{C}$, and then gradiently dehydrated 
with ethanol. After that, sections were embedded in epoxy resin Epon812 for overnight and cut in Leica DMIRB ultrathin microtome at $60-80 \mathrm{~nm}$ thickness, stained with uranyl acetate and lead citrate, and observed with a HC-1 $80.0 \mathrm{KV}$ Hitachi TEM system (Analytical \& Testing Center, IHB, CAS).

\section{RNA extraction, sequencing, assembly and functional annotation of liver transcriptomes}

Three liver samples of two groups, normal liver and fatty liver, were listed as biological replicates (NL-1, NL-2, NL3 and FL-1, FL-2, FL-3). Total RNA was extracted with RNeasy Mini Kit (Qiagen, Beijing) according to the manufacture's protocols. Proper quality and quantity were checked with Nanodrop ${ }^{\odot}$ ND-2000 spectrophotometer (LabTech, USA) and Technologies 2100 Bioanalyzer (Agilent Tech, USA) by measuring the $260 / 280 \mathrm{~nm}$ absorbance ratio. Fifty $\mu$ g total RNA of each samples were used for cDNA library establishing and high-throughput sequencing via BGISEQ-500 platform in BGI Genomics Co., Ltd., Shenzhen, China. Clean reads were mapped to the gibel carp' genome and genes using HISAT (v2.1.0) [82] and Bowtie2(v2.2.5) [83] (unpublished data). The expression levels of transcripts were calculated with RSEM [84]. Pearson's correlation coefficient $(\mathrm{r})$ and PCA were measured by using cor \& princomp in R for the strength of the association between the two groups. DEG analysis was conducted by DEseq2 $[85,86]$ with q-value (adjusted $p$ value $\leq 0.05$. GO and KEGG pathway enrichment analysis were conducted with phyper in R. $p$-value was corrected with false discovery rate (FDR) cut-off of 0.01 and q-value $\leq 0.05$ was used as the threshold to judge the significance of GO or KEGG enrichment. The raw sequences were deposited into the NCBI Sequence Read Archive (SRA) database (Accessions PRJNA675741).

\section{Latent pathways network construction and the significant pathways identification}

Latent pathway identification analysis, LPIA, developed by Pham et al., is a method to identify the interactions of pathways associated with DEGs and biological processes $[34,64]$. After preparing the three distinct but interrelated sources of biological pathways (such as "KEGG", P), biological functions (such as "GO", G) and gene transcriptional response in different conditions (such as "DEGs", DE) (Table S4), 1) First, we constructed a P-G bipartite graph if $\mathrm{P}$ and $\mathrm{G}$ shares a non-empty intersection of $\mathrm{DE}$, we calculated the weighted edge, denoted $\mathrm{W}_{\mathrm{GP}}$, which stands for the intersection of P and G as follow

$$
\mathrm{W}_{G P}=(|\mathrm{G} \cap \mathrm{P}|) /(|\mathrm{G} \cup \mathrm{P}|) \times \operatorname{median}\left\{\mathrm{DE}_{x} ; x \in \mathrm{G} \cap \mathrm{P}\right\}
$$

2) And then, converted the P-G bipartite graph to P-P pathway network if two pathways, $\mathrm{P}_{\mathrm{i}}$ and $\mathrm{P}_{\mathrm{j}}$ shares non- empty intersection of $G$, the weight between $P_{i}$ and $P_{j}$, denoted $A_{i j}$ is

$$
A_{i j}=\sum_{k}^{G} W_{G_{k} p_{i}} \times W_{G_{k} p_{j}}
$$

3) Finally, measured pathway importance via eigenvector centrality to determine the significance of a node in the above network after proper iterative operation. The weight scores $>0.1$ were used to draw P-P pathway network with cytoscape 3.7.2 [87]. The whole process was repeated using the bootstrap method [88] and the adjusted $p$-values were calculated to account for multiple testing with methods described by Dudoit and van der Laan [89].

\section{Quantitative reverse transcription PCR (qPCR)}

The first-strand cDNA was synthesized from total RNA following the protocol of Thermo Scientific ${ }^{\mathrm{TM}}$ RevertAid First Strand cDNA Synthesis Kit (Thermo Fisher Scientific, USA) in a $20 \mu$ reaction volume. The expression level was analyzed on a CFX96 ${ }^{\text {тм }}$ Real-Time PCR System (Bio-Rad, USA) using an iTaqTM Universal SYBR ${ }^{\circ}$ Green Supermix (Bio-Rad, USA). Gene-specific primers (Table S8) were designed with Primer premier 5.0 [90]. The reaction system, protocol, and endogenous control selection were conducted as previously described [91-93]. All liver samples were analyzed with three biological replicates and the relative expression levels of target genes were normalized to $\beta$-actin and calculated by the $2^{-\Delta \Delta C T}$ method. $p$-value $<0.05$ was considered statistically significant.

\begin{abstract}
Abbreviations
AMPK: AMP-activated protein kinase; acs/4: Acyl-CoA synthetase long-chain family member 4; BP: Biological processes; CC: Cellular component;

DEG: Differential gene expression; FBW: The final body weight; FDR: False discovery rate; GO: Gene Ontology; gcl: Glutamate-cysteine ligase; gpx4: Glutathione peroxidase 4; HE: Hematoxylin \& eosin; NAFL

D: Nonalcoholic fatty liver disease; JNK: C-jun Nterminal kinase; LPIA: Latent pathway identification analysis; KEGG: Kyoto Encyclopedia of Genes and Genomes; MF: Molecular functions; Myd88: Myeloid differentiation primaryresponse protein 88; NF-kB: Nuclear factor KB; Nrf2: Nuclear erythroid 2related factor 2; OCT: Optimum cutting temperature; ORO: Oil red o; PCA: Principal component analysis; PFA: Paraformaldehyde; qPCR: Quantitative reverse transcription PCR; ROS: Reactive oxygen species; SGR: Specific growth rate; TFEB: Transcription factor EB; tfr1: Transferrin receptor 1; TLRs: Toll-like receptors; Torc1: Target-of-rapamycin complex 1
\end{abstract}

\section{Supplementary Information}

The online version contains supplementary material available at https://doi. org/10.1186/s12864-021-07621-2.

Additional file 1: Figure S1 PCA analysis and relevant heat map of six liver samples. a PCA plot of DEGs among liver samples. Yellow circle: samples in PC1 belong to fatty liver. Blue circle: samples in PC2 belong to normal liver. b Relevant heat map of six liver samples. NL: normal liver, FL: fatty liver. 
Additional file 2: Table S1 DEGs (FDR $\leq 0.001)$ in normal liver and fatty liver. Lists of DEGs include Gene ID, Length, FPKM, Log fold change, Qvalue, $P$ - value, and Annotation.

Additional file 3: Table S2 GO terms with all up/downregulated DEGs enrichment analysis. GO terms with all upregulated DEGs (GO terms with all up-DEGs), GO enrichment analysis with all upregulated DEGs (enriched GO-up), GO terms with all downregulated DEGs (GO terms with all down-DEGs) and GO enrichment analysis with all downregulated DEGs (enriched GO-down). NL: normal liver, FL: fatty liver.

Additional file 4: Table S3 KEGG pathway enrichment analysis. Pathway ID, Pathway Name, KEGG function classification, number of Candidate and total DEGs/genes, Rich Ratio, $P$-value, Q-value are shown. $\mathrm{NL}$ : normal liver, FL: fatty liver.

Additional file 5: Figure S2 The left three latent pathways. a Porphyrin and chlorophyll metabolism pathway (23 DEGs, ko00860, https://www. genome.jp/dbget-bin/www_bget?map00860). b HIF-1 signaling pathway (49 DEGs, ko04066, https://www.kegg.jp/kegg-bin/show pathway?map05211). c Mineral absorption pathway (17 DEGs, ko04978, https://www.genome.jp/dbget-bin/www_bget?map04978) in comparative transcriptomic analysis. Up and downregulated DEGs are shown in red and green, respectively.

Additional file 6: Table S4 Input and output files in LPIA. Biological pathways (FL_NL_P), biological functions (FL_NL_G) and gene transcriptional response in different conditions (FL_NL_DE) are used in LPIA and the output file (Output_LPIA_FL_NL). NL: normal liver, FL: fatty liver.

Additional file 7: Table S5 Pathway-pathway network. The pathways include 43 enriched pathways in KEGG enrichment analysis and four significant pathways in LPIA method.

Additional file 8: Table S6 Growth performance of gibel carp in the Luo-Fu Lotus Farm. Lists of parameters include: initial body weight (IBW), final body weight (FBW) and specific growth rate (SGR).

Additional file 9: Table S7 Aquaculture conditions in the Luo-Fu Lotus Farm. Lists of conditions include Water temperature (WT), dissolved oxygen $(\mathrm{DO})$, total nitrogen $(\mathrm{TN})$, ammonia $\left(\mathrm{NH}_{4}-\mathrm{N}\right)$, nitrate $\left(\mathrm{NO}_{3}-\mathrm{N}\right)$, nitrite $\left(\mathrm{NO}_{2}-\mathrm{N}\right)$, and total phosphorus (TP).

Additional file 10: Checklist S1 Completed ARRIVE guidelines checklist. The checklist includes the ARRIVE Essential 10 and the Recommended Set.

Additional file 11: Table S8 Primers used in this study.

\section{Acknowledgments}

We would like to thank Dr. Xiao Yuan at The Analysis and Testing Center of Institute of Hydrobiology, Chinese Academy of Sciences for her assistance with electron microscope analysis. The research was supported by the Wuhan Branch, Supercomputing Center, Chinese Academy of Sciences, China.

\section{Authors' contributions}

XJZ performed laboratory experiments, analyzed the data and drafted the manuscript. LZ designed the studies and revised manuscript. WJL, WXD, XYL, ZWW and ZL participated in the laboratory experiments. YW participated in the data analysis. XYM and MD participated in the aquaculture conditions analysis. JFG conceived the studies and revised the manuscript. All authors have read and approved the manuscript.

\section{Funding}

This work was supported by the National Key Research and Development Project (2018YFD0901701), the earmarked fund for China Agriculture Research System (CARS-45-07), the Autonomous Project of the State Key Laboratory of Freshwater Ecology and Biotechnology (2019FBZ04), and the Autonomous Project of the Institute of Hydrobiology, Chinese Academy of Sciences (Y45A171).

\section{Availability of data and materials}

The raw sequences supporting the conclusions of this article were deposited into the NCBI Sequence Read Archive (SRA) database under the accession number PRJNA675741(NL: SRR13023909, SRR13023907 and SRR13023906; FL: SRR13023911, SRR13023910 and SRR13023908).

\section{Declarations}

\section{Ethics approval and consent to participate}

All procedures in this study were performed with the approval of Animal Care and Use Committee of Institute of Hydrobiology, Chinese Academy of Sciences (IHB, CAS, Protocol No. 2016-018).

\section{Consent for publication}

Not applicable.

\section{Competing interests}

The authors declare that they have no competing interests.

\section{Author details}

'College of Fisheries, Huazhong Agricultural University, Wuhan 430070, China. ${ }^{2}$ State Key Laboratory of Freshwater Ecology and Biotechnology, Institute of Hydrobiology, Innovation Academy for Seed Design, Chinese Academy of Sciences, Wuhan 430072, Hubei, China. ${ }^{3}$ University of Chinese Academy of Sciences, Beijing 100049, China.

Received: 24 December 2020 Accepted: 14 April 2021

Published online: 05 May 2021

\section{References}

1. Du ZY. Causes of fatty liver in farmed fish:a review and new perspectives (in Chinese). J Fish China. 2014;38(9):1628-38.

2. Zhao HH, Chong J, Tang R, Li L, Xia JG, Li DP. Metabolomics investigation of dietary effects on flesh quality in grass carp (Ctenopharyngodon idellus). Gigascience. 2018;7(10):giy111.

3. The Food and Agriculture Organization Fisheries Division. Cultured aquatic species information programme (CASIP) [online: http://www.fao.org/fishery/ culturedspecies/search/en]. Rome.

4. He S, Li L, Lv LY, Cai WJ, Dou YQ, Li J, et al. Mandarin fish (Sinipercidae) genomes provide insights into innate predatory feeding. Commun Biol. 2020;3(1):361. https://doi.org/10.1038/s42003-020-1094-y.

5. Han D, Shan XJ, Zhang WB, Chen YS, Wang QY, Li ZJ, et al. A revisit to fishmeal usage and associated consequences in Chinese aquaculture. Rev Aquacul. 2018;10(2):493-507. https://doi.org/10.1111/raq.12183.

6. Francis G, Makkar HPS, Becker K. Antinutritional factors present in plantderived alternate fish feed ingredients and their effects in fish. Aquaculture. 2001;199(3-4):197-227. https://doi.org/10.1016/S0044-8486(01)00526-9.

7. Zhao H, Xia J, Zhang X, He X, Li L, Tang R, et al. Diet affects muscle quality and growth traits of grass carp (Ctenopharyngodon idellus): a comparison between grass and artificial feed. Front Physiol. 2018;9:283-95. https://doi. org/10.3389/fphys.2018.00283.

8. Kolkovski S. Digestive enzymes in fish larvae and juveniles - implications and applications to formulated diets. Aquaculture. 2001;200(1-2):181-201. https://doi.org/10.1016/S0044-8486(01)00700-1.

9. Oyarzun R, Martinez D, Soto-Davila M, Munoz JLP, Dantagnan P, VargasChacoff $L$. Effect of ration level on growth performance, body composition, intermediary metabolism and serum parameters in juvenile Patagonian blennie Eleginops maclovinus. Comp Biochem Physiol A Mol Integr Physiol. 2019;230:122-30. https://doi.org/10.1016/j.cbpa.2019.01.013.

10. Spisni E, Tugnoli M, Ponticelli A, Mordenti T, Tomasi V. Hepatic steatosis in artificially fed marine teleosts. J Fish Dis. 1998;21(3):177-84. https://doi.org/1 0.1046/j.1365-2761.1998.00089.x.

11. Jia R, Cao LP, Du JL, He Q, Gu ZY, Jeney G, et al. Effects of high-fat diet on steatosis, endoplasmic reticulum stress and autophagy in liver of tilapia (Oreochromis niloticus). Front Mar Sci. 2020;7:363-76. https://doi.org/10.3389/ fmars.2020.00363.

12. Jia R, Cao LP, Du JL, He Q, Gu ZY, Jeney G, et al. Effects of high-fat diet on antioxidative status, apoptosis and inflammation in liver of tilapia (Oreochromis niloticus) via Nrf2, TLRs and JNK pathways. Fish Shellfish Immun. 2020;104:391-401. https://doi.org/10.1016/j.fsi.2020.06.025

13. Sapp V, Gaffney L, EauClaire SF, Matthews RP. Fructose leads to hepatic steatosis in zebrafish that is reversed by mechanistic target of rapamycin (mTOR) inhibition. Hepatology. 2014;60(5):1581-92. https://doi.org/10.1002/ hep.27284. 
14. Huang LD, Cheng Y, Huang K, Zhou Y, Ma YQ, Zhang MC. Ameliorative effect of Sedum sarmentosum Bunge extract on Tilapia fatty liver via the PPAR and P53 signaling pathway. Sci Rep-Uk. 2018;8:11.

15. Tao YF, Qiang J, He J, Zhu H-J, Bao JW, Xu P. Untargeted LC-MS metabolomics approach reveals metabolic changes in genetically improved farmed tilapia (Oreochromis niloticus) with fatty liver induced by a high-fat diet. Aquac Res. 2020; doi.org/10.1111/are.14929.

16. Tian J, Lu X, Jiang M, Wu F, Liu W, Yu LJ, et al. AMPK activation by dietary AICAR affects the growth performance and glucose and lipid metabolism in juvenile grass carp. Aquac Nutr. 2020;26(1):3-14. https://doi.org/10.1111/a nu.12951.

17. Zhang Y, Chen P, Liang XF, Han J, Wu XF, Yang YH, et al. Metabolic disorder induces fatty liver in Japanese seabass, Lateolabrax japonicas fed a full plant protein diet and regulated by CAMP-JNK/NF-kB-caspase signal pathway. Fish Shellfish Immun. 2019;90:223-34. https://doi.org/10.1016/j.fsi.2019.04.060.

18. Gui JF, Zhou L. Genetic basis and breeding application of clonal diversity and dual reproduction modes in polyploid Carassius auratus gibelio. Sci China Life Sci. 2010;53(4):409-15. https://doi.org/10.1007/s11427-010-0092-6.

19. Wang ZW, Zhu HP, Wang D, Jiang FF, Guo W, Zhou L, et al. A novel nucleocytoplasmic hybrid clone formed via androgenesis in polyploid gibel carp. BMC Res Notes. 2011;4(82):1-13.

20. Zhou L, Gui JF. Natural and artificial polyploids in aquaculture. Aquacult Fish. 2017;2(3):103-11. https://doi.org/10.1016/j.aaf.2017.04.003.

21. Gui JF, Tang QS, Li ZJ, Liu JS, De Silver SS. Aquaculture in China: success stories and modern trends. Oxford: Wiley; 2018. https://doi.org/10.1002/ 9781119120759.

22. Li XY, Liu XL, Zhu YJ, Zhang J, Ding M, Wang MT, et al. Origin and transition of sex determination mechanisms in a gynogenetic hexaploid fish. Heredity (Edinb). 2018;121(1):64-74. https://doi.org/10.1038/s41437-017-0049-7.

23. Chen F, Li XY, Zhou L, Yu P, Wang ZW, Li Z, et al. Stable genome incorporation of sperm-derived DNA fragments in gynogenetic clone of gibel carp. Mar Biotechnol (NY). 2020;22(1):54-66. https://doi.org/10.1007/s1 0126-019-09930-w.

24. Bureau of Fisheries of MOA. National fisheries Technology Extension Center China Society of Fisheries. China fisheries statistical yearbook 2020 (in Chinese). Beijing: China Agriculture Press; 2020.

25. Mortazavi A, Williams BA, Mccue K, Schaeffer L, Wold B. Mapping and quantifying mammalian transcriptomes by RNA-Seq. Nat Methods. 2008;5(7): 621-8. https://doi.org/10.1038/nmeth.1226.

26. Conesa A, Madrigal P, Tarazona S, Gomez-Cabrero D, Cervera A, McPherson A, et al. A survey of best practices for RNA-seq data analysis. Genome Biol. 2016;17(1):13-31. https://doi.org/10.1186/s13059-016-0881-8.

27. Hyslop PA, Hinshaw DB, Halsey WA, Schraufstatter IU Jr, Sauerheber RD, Spragg RG, et al. Mechanisms of oxidant-mediated cell injury. The glycolytic and mitochondrial pathways of ADP phosphorylation are major intracellular targets inactivated by hydrogen peroxide. J Biol Chem. 1988;263(4):1665-75. https://doi.org/10.1016/S0021-9258(19)77928-9.

28. Abe T, Hashimoto Y, Hosaka H, Tomita-Yokotani K, Kobayashi M. Discovery of amide (peptide) bond synthetic activity in acyl-CoA synthetase. J Biol Chem. 2008;283(17):11312-21. https://doi.org/10.1074/jbc.M709654200.

29. Kerner J, Hoppel C. Fatty acid import into mitochondria. BBA-Mol Cell Biol L. 2000;1486(1):1-17. https://doi.org/10.1016/\$1388-1981(00)00044-5.

30. Tanaka K. The proteasome: overview of structure and functions. Proc Jpn Acad Ser B Phys Biol Sci. 2009;85(1):12-36. https://doi.org/10.2183/pjab.85.12.

31. Kanehisa M, Furumichi M, Sato $Y$, Ishiguro-Watanabe M, Tanabe M. KEGG: integrating viruses and cellular organisms. Nucleic Acids Res. 2021;49:545-51.

32. Xie $Y$, Hou W, Song $X, Y u Y$, Huang J, Sun $X$, et al. Ferroptosis: process and function. Cell Death Differ. 2016;23(3):369-79. https://doi.org/10.1038/cdd.2 015.158.

33. Yang WS, Stockwell BR. Ferroptosis: death by lipid peroxidation. Trends Cell Biol. 2016;26(3):165-76. https://doi.org/10.1016/j.tcb.2015.10.014.

34. Pham L, Christadore L, Schaus S, Kolaczyk ED. Network-based prediction for sources of transcriptional dysregulation using latent pathway identification analysis. Proc Natl Acad Sci U S A. 2011;108(32):13347-52. https://doi.org/1 0.1073/pnas.1100891108

35. Forcina GC, Dixon SJ. GPX4 at the crossroads of lipid homeostasis and ferroptosis. Proteomics. 2019;19(18):e1800311. https://doi.org/10.1002/pmic.2 01800311.

36. Yang WRS, SriRamaratnam R, Welsch ME, Shimada K, Skouta R, Viswanathan VS, et al. Regulation of ferroptotic cancer cell death by GPX4. Cell. 2014; 156(1-2):317-31. https://doi.org/10.1016/j.cell.2013.12.010.
37. Angeli JPF, Schneider M, Proneth B, Tyurina YY, Tyurin VA, Hammond VJ, et al. Inactivation of the ferroptosis regulator Gpx4 triggers acute renal failure in mice. Nat Cell Biol. 2014;16(12):1180-91. https://doi.org/10.1038/ ncb3064.

38. Capelletti MM, Manceau H, Puy H, Peoc'h K. Ferroptosis in liver diseases: an overview. Int J Mol Sci. 2020;21(14):4908-30. https://doi.org/10.3390/ ijms21144908.

39. Bersuker K, Hendricks JM, Li ZP, Magtanong L, Ford B, Tang PH, et al. The CoQ oxidoreductase FSP1 acts parallel to GPX4 to inhibit ferroptosis. Nature. 2019;575(7784):688-92. https://doi.org/10.1038/s41586-019-1705-2.

40. Conrad M, Kagan VE, Bayir H, Pagnussat GC, Head B, Traber MG, et al. Regulation of lipid peroxidation and ferroptosis in diverse species. Genes Dev. 2018;32(9-10):602-19. https://doi.org/10.1101/gad.314674.118.

41. Doll S, Proneth B, Tyurina YY, Panzilius E, Kobayashi S, Ingold I, et al. ACSL4 dictates ferroptosis sensitivity by shaping cellular lipid composition. Nat Chem Biol. 2017;13(1):91-8. https://doi.org/10.1038/nchembio.2239.

42. Yuan $\mathrm{H}$, Li XM, Zhang XY, Kang R, Tang DL. Identification of ACSL4 as a biomarker and contributor of ferroptosis. Biochem Biophys Res Commun. 2016;478(3):1338-43. https://doi.org/10.1016/j.bbrc.2016.08.124.

43. Feng H, Schorpp K, Jin J, Yozwiak CE, Hoffstrom BG, Decker AM, et al. Transferrin receptor is a specific ferroptosis marker. Cell Rep. 2020;30(10): 3411-23. https://doi.org/10.1016/j.celrep.2020.02.049.

44. Nishizawa S, Araki H, Ishikawa Y, Kitazawa S, Hata A, Soga T, et al. Low tumor glutathione level as a sensitivity marker for glutamate-cysteine ligase inhibitors. Oncol Lett. 2018;15(6):8735-43. https://doi.org/10.3892/ol.2018. 8447

45. Gao MH, Yi JM, Zhu JJ, Minikes AM, Monian P, Thompson CB, et al. Role of mitochondria in ferroptosis. Mol Cell. 2019;73(2):354-63. https://doi.org/10.1 016/j.molcel.2018.10.042.

46. Schafer FQ, Buettner GR. Acidic pH amplifies iron-mediated lipid peroxidation in cells. Free Radical Bio Med. 2000;28(8):1175-81. https://doi. org/10.1016/S0891-5849(00)00319-1.

47. Lin YR, Huang SL, Huang $\mathrm{CH}$. Characteristics of NADH-dependent lipid peroxidation in sarcoplasmic reticulum of white shrimp, Litopenaeus vannamei, and freshwater prawn, Macrobrachium rosenbergii. Comp Biochem Phys B. 2003;135(4):683-7. https://doi.org/10.1016/S1096-4959(03 )00149-0.

48. Tanaka $R$, Higo $Y$, Murata $H$, Nakamura T. Accumulation of hydroxy lipids in live fish with oxidative stress. Fish Sci. 1999;65(5):796-7. https://doi.org/1 0.2331/fishsci.65.796.

49. Tanaka R. Lipid peroxide levels and accumulation of hydroxy lipids in live fish with oxidative stress. J Natl Fish Univ. 2007;56(1):107-18.

50. Ren C, Hu X, Li X, Zhou Q. Ultra-trace graphene oxide in a water environment triggers Parkinson's disease-like symptoms and metabolic disturbance in zebrafish larvae. Biomaterials. 2016;93:83-94. https://doi.org/1 0.1016/j.biomaterials.2016.03.036

51. Griffin A, Parajes S, Weger M, Zaucker A, Taylor AE, O'Neil DM, et al. Ferredoxin $1 b(F d x 1 b)$ is the essential mitochondrial redox partner for cortisol biosynthesis in zebrafish. Endocrinology. 2016;157(3):1122-34. https://doi.org/10.1210/en.2015-1480.

52. Du ZY, Clouet $P$, Huang LM, Degrace $P$, Zheng WH, He JG, et al. Utilization of different dietary lipid sources at high level in herbivorous grass carp (Ctenopharyngodon idella): mechanism related to hepatic fatty acid oxidation. Aquac Nutr. 2008;14(1):77-92. https://doi.org/10.1111/j.1365-2095.2007.00507.x.

53. Dixon SJ, Lemberg KM, Lamprecht MR, Skouta R, Zaitsev EM, Gleason CE, et al. Ferroptosis: an iron-dependent form of nonapoptotic cell death. Cell. 2012;149(5):1060-72. https://doi.org/10.1016/j.cell.2012.03.042.

54. Nirmala JG, Lopus M. Cell death mechanisms in eukaryotes. Cell Biol Toxicol. 2020;36(2):145-64. https://doi.org/10.1007/s10565-019-09496-2.

55. Tsurusaki S, Tsuchiya Y, Koumura T, Nakasone M, Sakamoto T, Matsuoka M, et al. Hepatic ferroptosis plays an important role as the trigger for initiating inflammation in nonalcoholic steatohepatitis. Cell Death Dis. 2019;10(6):44962. https://doi.org/10.1038/s41419-019-1678-y.

56. McDougall M, Choi J, Kim HK, Bobe G, Stevens JF, Cadenas E, et al. Lethal dysregulation of energy metabolism during embryonic vitamin E deficiency. Free Radic Biol Med. 2017;104:324-32. https://doi.org/10.1016/j.freera dbiomed.2017.01.020.

57. Saetan W, Tian C, Yu J, Lin X, He F, Huang Y, et al. Comparative Transcriptome analysis of gill tissue in response to hypoxia in Silver Sillago (Sillago sihama). Animals. 2020;10(4):628-40. https://doi.org/10.33 90/ani10040628. 
58. Zhou T, Gui L, Liu M, Li W, Hu P, Duarte DFC, et al. Transcriptomic responses to low temperature stress in the Nile tilapia, Oreochromis niloticus. Fish Shellfish Immun. 2019;84:1145-56. https://doi.org/10.1016/j.fsi.2018.10.023.

59. Sun Z, Gong C, Ren J, Zhang X, Wang G, Liu Y, et al. Toxicity of nickel and cobalt in Japanese flounder. Environ Pollut. 2020;263:1-10.

60. Yang M, Lu Z, Li F, Shi F, Zhan F, Zhao L, et al. Escherichia coli induced ferroptosis in red blood cells of grass carp (Ctenopharyngodon idella). Fish Shellfish Immun. 2020; https://doi.org/10.1016/.jfsi.2020.09.036;112:159-67.

61. Kwon MY, Park E, Lee SJ, Chung SW. Heme oxygenase-1 accelerates erastininduced ferroptotic cell death. Oncotarget. 2015;6(27):24393-403. https:// doi.org/10.18632/oncotarget.5162.

62. Kong Z, Liu R, Cheng Y. Artesunate alleviates liver fibrosis by regulating ferroptosis signaling pathway. Biomed Pharmacother. 2019;109:2043-53. https://doi.org/10.1016/j.biopha.2018.11.030.

63. Wang H, Liu C, Zhao Y, Gao G. Mitochondria regulation in ferroptosis. Eur J Cell Biol. 2020;99(1):151058. https://doi.org/10.1016/j.ejcb.2019.151058.

64. Pham LM, Carvalho L, Schaus S, Kolaczyk ED. Perturbation detection through modeling of gene expression on a latent biological pathway network: a bayesian hierarchical approach. J Am Stat Assoc. 2016;111(513): 73-92. https://doi.org/10.1080/01621459.2015.1110523.

65. Bonkovsky HL, Guo JT, Hou WH, Li T, Narang T, Thapar M. Porphyrin and heme metabolism and the porphyrias. Compr Physiol. 2013;3(1):365-401. https://doi.org/10.1002/cphy.c120006.

66. Yao ZL, Wang H, Chen L, Zhou K, Ying CQ, Lai QF. Transcriptomic profiles of Japanese medaka (Oryzias latipes) in response to alkalinity stress. Genet Mol Res. 2012;11(3):2200-46. https://doi.org/10.4238/2012.June.15.2.

67. Xia MH, Bi K, Huang RL, Cho MH, Sakamuru S, Miller SC, et al. Identification of small molecule compounds that inhibit the HIF-1 signaling pathway. Mol Cancer. 2009;8(1):117-29. https://doi.org/10.1186/1476-4598-8-117.

68. Xiao W. The hypoxia signaling pathway and hypoxic adaptation in fishes. Sci China Life Sci. 2015;58(2):148-55. https://doi.org/10.1007/s11427-015-4 801-z.

69. Sarayloo F, Dionne-Laporte A, Catoire H, Rochefort D, Houle G, Ross JP, et al. Mineral absorption is an enriched pathway in a brain region of restless legs syndrome patients with reduced MEIS1 expression. PLoS One. 2019;14(11): e0225186. https://doi.org/10.1371/journal.pone.0225186.

70. Prabhu PAJ, Geurden I, Fontagne-Dicharry S, Veron V, Larroquet L, Mariojouls $\mathrm{C}$, et al. Responses in micro-mineral metabolism in rainbow trout to change in dietary ingredient composition and inclusion of a micromineral premix. PLoS One. 2016;11(2):e0149378. https://doi.org/10.1371/ journal.pone.0149378.

71. Qiu Y, Cao Y, Cao W, Jia Y, Lu N. The application of ferroptosis in diseases. Pharmacol Res. 2020;159:104919. https://doi.org/10.1016/j.phrs.2020.104919.

72. Dixon SJ, Patel D, Welsch M, Skouta R, Lee E, Hayano M, et al. Pharmacological inhibition of cystine-glutamate exchange induces endoplasmic reticulum stress and ferroptosis. Elife. 2014;3:02523. https:// doi.org/10.7554/eLife.02523.

73. Angeli JPF, Shah R, Pratt DA, Conrad M. Ferroptosis inhibition: mechanisms and opportunities. Trends Pharmacol Sci. 2017;38(5):489-98. https://doi. org/10.1016/j.tips.2017.02.005

74. Percie du Sert N, Hurst V, Ahluwalia A, Alam S, Avey MT, Baker M, et al. The ARRIVE guidelines 2.0: updated guidelines for reporting animal research. PLoS Biol. 2020. https://doi.org/10.1371/journal.pbio.3000410.

75. Xia W, Zhou L, Yao B, Li CJ, Gui JF. Differential and spermatogenic cellspecific expression of DMRT1 during sex reversal in protogynous hermaphroditic groupers. Mol Cell Endocrinol. 2007;263(1-2):156-72. https:// doi.org/10.1016/.mce.2006.09.014.

76. Zhu YJ, Li XY, Zhang J, Li Z, Ding M, Zhang XJ, et al. Distinct sperm nucleus behaviors between genotypic and temperature-dependent sex determination males are associated with replication and expression-related pathways in a gynogenetic fish. BMC Genomics. 2018;19(1):437-51. https:// doi.org/10.1186/s12864-018-4823-6

77. Ramirez-Zacarias JL, Castro-Munozledo F, Kuri-Harcuch W. Quantitation of adipose conversion and triglycerides by staining intracytoplasmic lipids with oil red O. Histochemistry. 1992;97(6):493-7. https://doi.org/10.1007/BF0031 6069.

78. Yu P, Wang Y, Yang WT, Li Z, Zhang XJ, Zhou L, Gui JF. Upregulation of the PPAR signaling pathway and accumulation of lipids are related to the morphological and structural transformation of the dragon-eye goldfish eye. Sci. China Life Sci. 2021; https://doi.org/10.1007/s11427-020-1814-1.
79. Mehlem A, Hagberg CE, Muhl L, Eriksson U, Falkevall A. Imaging of neutral lipids by oil red $\mathrm{O}$ for analyzing the metabolic status in health and disease. Nat Protoc. 2013;8(6):1149-54. https://doi.org/10.1038/nprot.2013.055.

80. Kim YS, Ke F, Lei XY, Zhu R, Zhang QY. Viral envelope protein 53R gene highly specific silencing and iridovirus resistance in fish cells by amiRNA. PLoS One. 2010;5(4):e10308. https://doi.org/10.1371/journal.pone.0010308.

81. Gao EB, Gui JF, Zhang QY. A novel cyanophage with a cyanobacterial nonbleaching protein a gene in the genome. J Virol. 2012;86(1):236-45. https://doi.org/10.1128/JVI.06282-11.

82. Kim D, Langmead B, Salzberg SL. HISAT: a fast spliced aligner with low memory requirements. Nat Methods. 2015;12(4):357-60. https://doi.org/10.1 038/nmeth.3317.

83. Langmead B, Salzberg SL. Fast gapped-read alignment with bowtie 2. Nat Methods. 2012;9(4):357-9. https://doi.org/10.1038/nmeth.1923.

84. Li B, Dewey CN. RSEM: accurate transcript quantification from RNA-Seq data with or without a reference genome. BMC Bioinform. 2011;12(1):323-38. https://doi.org/10.1186/1471-2105-12-323.

85. Love Ml, Huber W, Anders S. Moderated estimation of fold change and dispersion for RNA-seq data with DESeq 2. Genome Biol. 2014;15(12):55070. https://doi.org/10.1186/s13059-014-0550-8.

86. Anders $\mathrm{S}$, Huber W. Differential expression analysis for sequence count data. Genome Biol. 2010;11(10):R106-17. https://doi.org/10.1186/gb-2010-11-10-r1 06.

87. Shannon P, Markiel A, Ozier O, Baliga NS, Wang JT, Ramage D, et al. Cytoscape: a software environment for integrated models of biomolecular interaction networks. Genome Res. 2003;13(11):2498-504. https://doi.org/1 $0.1101 /$ gr. 1239303

88. Kim JH. BootPR: Boostrap prediction intervals and bias-corrected forecasting 2009. [online: https://rdrr.io/cran/BootPR/].

89. Dudoit S, Laan M. Multiple testing procedures with applications to genomics. New York: Springer; 2008.

90. Singh VK, Mangalam AK, Dwivedi S, Naik S. Primer premier: program for design of degenerate primers from a protein sequence. Biotechniques. 1998;24(2):318-9. https://doi.org/10.2144/98242pf02.

91. Gao FX, Wang Y, Zhang QY, Mou CY, Li Z, Deng YS, et al. Distinct herpesvirus resistances and immune responses of three gynogenetic clones of gibel carp revealed by comprehensive transcriptomes. BMC Genomics. 2017;18(1):561-79. https://doi.org/10.1186/s12864-017-3945-6.

92. Mou CY, Wang Y, Zhang QY, Gao FX, Li Z, Tong JF, Zhou L, Gui JF. Differential interferon system gene expression profiles in susceptible and resistant gynogenetic clones of gibel carp. Dev Comp Immunol. 2018;86:5264. https://doi.org/10.1016/j.dci.2018.04.024.

93. Lu WJ, Gao FX, Wang Y, Zhang QY, Li Z, Zhang XJ, et al. Differential expression of innate and adaptive immune genes in the survivors of three gibel carp gynogenetic clones after herpesvirus challenge. BMC Genomics. 2019;20(1):432-50. https://doi.org/10.1186/s12864-019-5777-z.

\section{Publisher's Note}

Springer Nature remains neutral with regard to jurisdictional claims in published maps and institutional affiliations.

Ready to submit your research? Choose BMC and benefit from:

- fast, convenient online submission

- thorough peer review by experienced researchers in your field

- rapid publication on acceptance

- support for research data, including large and complex data types

- gold Open Access which fosters wider collaboration and increased citations

- maximum visibility for your research: over $100 \mathrm{M}$ website views per year

At BMC, research is always in progress.

Learn more biomedcentral.com/submissions 